\title{
Autosomal recessive palmoplantar keratoderma and congenital alopecia
}

INSERM

\section{Source}

INSERM. (1999). Orphanet: an online rare disease and orphan drug data base. Autosomal recessive palmoplantar keratoderma and congenital alopecia. ORPHA:1366

Autosomal recessive palmoplantar hyperkeratosis and congenital alopecia (PPK-CA) is a rare genetic skin disorder characterized by cong enital alopecia and palmoplantar hyperkeratosis. It is usually associated with cataracts, progressive sclerodactyly and pseudo-ainhum. 